\title{
Newborn Resuscitation Scale Up and Retention Program Associated with Improved Neonatal Outcomes in Western Nepal
}

\author{
Naresh Pratap KC ${ }^{1}$, Ranjan Dhungana ${ }^{1}$, Emily Gamboa ${ }^{2}$, Siena F Davis ${ }^{2}$, Michael $K$ Visick ${ }^{3}$ and Robert \\ B Clark, MD, MPH ${ }^{*}$ (iD)
}

${ }^{1}$ Safa Sanaulo Nepal, Kathmandu, Nepal

${ }^{2}$ Children's Medical Mission, Salem, USA

${ }^{3}$ University of Utah School of Medicine, Salt Lake City, USA

${ }^{4}$ Brigham Young University, Provo, USA

*Corresponding author: Robert B Clark, MD, MPH, Brigham Young University, Provo, Utah, USA

\begin{abstract}
Background: The adoption of the Helping Babies Breath (HBB) tool has improved the outcomes of neonatal resuscitation following intrapartum events. Perinatal asphyxia however remains a leading cause of neonatal morbidity and mortality in Nepal. HBB training has proven effective, but a major challenge is maintaining resuscitation skills over time. Safa Sanaulo Nepal (SSN) designed an evidence-based strategy for scaling up training and sustaining long-term retention. This paper describes the implementation of SSN's model, and changes in newborn outcomes that occurred during the program.

Methods and findings: The skills retention strategy relied on facility-based trainers to scale up and maintain resuscitation skills in 18 facilities in the area of Nepalgunj, Nepal. A single external mentor coached and assisted the facilitybased trainers, provided general support, and monitored progress. Prospective outcome monitoring tracked changes in health metrics for a period of 24 months (March 2018 to March 2020). During this time, 46 facility-based trainers taught resuscitation skills to 1,785 midwives, nurses, and physicians, and supported skill retention with limited data gathered on neonatal health outcomes of 49,809 vaginal deliveries and 12,823 Caesarean sections. To analyze changes over the time SSN's program was implemented, a comparison of beginning (first three months of assessment) and follow-up (last three months) rates of neonatal mortality, morbidity, and stillbirths was conducted. The total number of births assessed in this comparison was 15,947.
\end{abstract}

\begin{abstract}
Results indicate mortality dropped $60 \%(p=0.01)$, morbidity dropped $77 \%(p=0.01)$, and intrapartum stillbirths dropped $73 \%(p=0.001)$ from beginning to follow-up.

Conclusions: SSN's model provides a valuable example of how an evidence-based program focusing on facilitybased trainers, who are mentored and supported to scaleup and sustain resuscitation skills over time, may have a substantial influence on critical neonatal outcomes. This program demonstrated that capacity building required minimal external support and expense, with a single mentor mentoring, supporting, and monitoring 18 facilities. Future programs working to reduce neonatal mortality, morbidity, and intrapartum stillbirths may incorporate program elements to further improve neonatal outcomes.
\end{abstract}

\section{Introduction}

The Millennium Development Goals (MDG) brought focus and resources to child and newborn outcomes worldwide. In Nepal, the three goals of MDG Target \# 4 (Reducing Child Mortality) were met. However, while the under-5 mortality rate (U5MR) did decline, major inequalities persist in parts of Nepal [1]. The neonatal mortality rate (NMR) reduction did not decline as rapidly as the U5MR, causing the NMR to rise to $61 \%$ of the U5MR [2].

Intrapartum events leading to asphyxia around the time of birth continue to be a leading cause of neonatal

\footnotetext{
Citation: Naresh PKC, Dhungana R, Gamboa E, Davis SF, Visick MK, et al. (2022) Newborn Resuscitation Scale Up and Retention Program Associated with Improved Neonatal Outcomes in Western Nepal. Int J Pediatr Res 8:087. doi.org/10.23937/2469-5769/1510087 Accepted: February 05, 2022: Published: February 07, 2022

Copyright: (C) 2022 Naresh PKC, et al. This is an open-access article distributed under the terms of the Creative Commons Attribution License, which permits unrestricted use, distribution, and reproduction in any medium, provided the original author and source are credited.
} 
morbidity and mortality in Nepal [3-6]. The Nepalese Ministry of Health and Population (MOHP) was an early adopter of programs to improve neonatal resuscitation in response to perinatal asphyxia, including the training of Female Community Health Volunteers (FCHV) in 2004 [7]. At that time the FCHV were supplied with bag and masks to support ventilation in order to address the burden of asphyxia. However, this intervention was not sustained.

In 2015, the Helping Babies Breathe (HBB) training curriculum, produced by the American Academy of Pediatrics, was incorporated into in-service training packages launched by the Child Health Division of the Ministry of Health and Population. An abbreviated form of HBB was specifically included in the Skill Birth Attendant in-service training package, and HBB training was encouraged in other settings. This decision was based upon, in part, the very successful pilot of HBB in Nepal in 2013 [8].

Multiple studies, including two systematic reviews, have documented HBB as an effective tool for reducing newborn morbidity and mortality, and reducing stillbirths [8-12]. The dissemination of improved resuscitation techniques using HBB has been supported by multiple child health partners in Nepal. UNICEF upgraded the resuscitation capacity in a portfolio of large hospitals during two clinical trials $[13,14]$. Safa Sanaulo Nepal (SSN), founded by the late Rakesh Hamal and supported by Latter-day Saint Charities (LDSC), has sponsored multiple HBB training-of-trainer (TOT) courses in all seven Nepal provinces since 2014. The National Health Training Center (NHTC), a branch of the MOHP, is responsible for disseminating approved in-service provider training at its centers throughout the country. NHTC has orchestrated HBB training in collaboration with LDSC and others [15].

HBB follow-up studies have shown a decline in knowledge and skills over time [16-19]. Thus, a major challenge in both clinical trials and implementation/ dissemination efforts around newborn resuscitation has been maintaining resuscitation skills over time. This dilemma has affected western Nepal as well as other regions and countries. The eight largest facilities in this program had previously received training in HBB but had failed to scale up and/or perpetuate the knowledge and skills of newborn resuscitation.

There is now a robust evidence base for effective tools to sustain the skills of resuscitation, including clinical trials conducted in Nepal [6,13,17,19,20-23]. These have provided guidance regarding the elements needed to both improve and retain resuscitation skills.

Sustainable change is further augmented by cost-effective strategies that include minimal data gathering and analysis. While well-funded, short-term clinical studies have produced important evidence for sustainable change, relatively little has been published regarding low-cost approaches to skill retention appropriate for low-to-middle-income countries (LMIC) [24]. In contrast, collection and analysis of minimal, easily obtainable indicators may enhance sustainability by decreasing the necessary resource input.

SSN designed a package centered on building and maintaining the capacity of facility-based trainers to sustain the skills required to manage newborn emergencies. The package had three fundamental goals:

1. Utilize evidence-based strategies for scaling up and supporting HBB

2. Implement a cost-effective program

3. Monitor the process with minimal indicators

The purpose of this paper is to describe the two-year implementation of SSN's package in western Nepal and report changes in newborn outcomes that occurred during the time of program implementation.

\section{Methods}

\section{Setting}

Nepalgunj is the third largest city in Province 5 of Nepal. Provinces 5, 6, and 7 comprise much of the former Mid-Western and Far-Western regions of Nepal, regions where child mortality and morbidity have traditionally been the highest [25]. As a sub-metropolitan city in the Terai region, Nepalgunj is a medical referral center for the surrounding districts and home to regional, subregional, and private healthcare facilities.

The Mid-Western and Far-Western Regions had been designated by NHTC as underserved by health services, with neonatal mortality above the national average. NHTC recommended specific facilities in these regions for support in training staff and increasing staff performance. Accordingly, a cross-section of both public and private facilities, including those with large delivery services, were invited to participate in a combined program of newborn resuscitation skills training, mentored scale-up, and on-going facility support.

\section{Program design}

In April 2018, the two-day HBBTOT course was attended by 46 trainers representing the 18 facilities in the study cohort. The training was held under NHTC direction and supervision.

The 46 trainers included 35 (76\%) nurses, 28 (61\%) who had worked in their facility for more than 5 years, and $19(42 \%)$ who worked in large urban hospitals. Twenty-four (53\%) indicated they personally attended up to 25 deliveries in an average month. Forty-two (93\%) trainers indicated they resuscitated babies with a bag and mask as part of their current job.

The HBB TOT was facilitated by eight experienced Nepali trainers and two U.S. trainers. SSN and LDSC provided the 46 new trainers with instructional 
materials (flipcharts, booklets and electronic copies), training mannequins (NeoNatalies), and delivery room equipment (bag-and-mask, Penguin suction, stethoscope) to facilitate immediate clinical and teaching applications in the 18 facilities.

Consistent with the goal of a low-cost training support program, a single experienced medical trainer with research experience was recruited to serve as the coordinator for the entire portfolio of facilities. As a full-time employee of SSN, the coordinator served as a trainer, mentor, administrative liaison, and monitor of health indicators.

The program coordinator utilized evidence-based methods to build the capacity of facility-based trainers and staff. The coordinator was empowered to:

- Scale-up HBB training to all relevant personnel, including new staff additions

- Promote low-dose, high-frequency practice, including daily bag-and-mask skill checks

- Provide refresher training

- Encourage structured self-evaluation

- Hold weekly or biweekly review meetings for peer review of the HBB protocol adherence

- Provide onsite coaching and mentoring

- Coordinate with administration

- Encourage staff communication and transparency via progress boards

The scale-up of resuscitation training and support activities followed the April 2018 TOT and continued for the next twenty-three months. Minimal key metrics of importance to NHTC were monitored to help measure the scale-up process and improvements in outcomes. The data collection was conducted according to Nepali months, comprising Chaitra 2074 through Falgun 2075, in accordance with MOHP convention (March 15, 2018 to March 14, 2020).

\section{Program monitoring and data collection}

Monthly training supervision reports generated by the program coordinator documented the scale-up and facility support, including the number of providers trained and retrained, maintenance of an equipped practice corner and low-dose high-frequency practice logs, completion of self-evaluation forms, review meetings held, and review of delivery and newborn logs.

Monthly facility-level metrics were also collected from each facility, including the number of vaginal deliveries, number of caesarean sections, number of intrapartum (fresh) and macerated stillbirths, number of neonatal deaths less than 24 hours, number of sick newborns transferred or discharged from the maternity unit.
In the tertiary level facilities, monthly facilitylevel data were derived from the health information department, which tabulated and reported MOHPmandated metrics. In smaller facilities, the program coordinator gathered data from registers or logs maintained in the labor room and in the maternity ward. Patient's personal data, such as demographic data, were not collected. In all facilities, data was customarily curated and reported by the month and year of the Nepali calendar.

Data was collected from the Nepali month and year of Chaitra 2074 to Chaitra 2076, for a total of 25 Nepali months. Trends were assessed over the entire 25 -month period. To better understand improvements in care, the initial three months of data were compared with the final three months of data. The initial three months of Ashad, Shrawan, and Bhadra 2075 included one month before training began and the first two months after the training of trainers, during which time scale-up was beginning. The final three months of Poush, Magh, and Falgun 2076 comprised the time in which the training had previously been scaled up, with mentoring and supportive mechanisms well established.

\section{Data analysis}

The monthly scale-up and supportive supervision parameters were generally qualitative in nature, with a descriptive summary provided below.

Facility-level metrics were analyzed, including the total number of deliveries, Caesarian sections, neonatal deaths less than 24 hours, intrapartum still births, and sick newborns transferred or discharged. The beginning measurements were the sum of these data for the initial three months and the follow-up measurements were the sum of data from the final three months. A Paired T-Test was conducted to determine if beginning and follow-up values were significantly different. The number of deliveries and Caesarian sections were analyzed as potential confounding factors.

The program coordinator collected data from standard hospital reports and hospital logs into monthly reports, with the program coordinator responsible for data quality checks and integrity. The monthly reports were forwarded to SSN and to US-based research assistants. SSN conducted an initial review, analysis, and prepared mandated reports. Research assistants combined the data into a master file, clarified missing variables with the coordinator, deleted variables missing significant data, changed text into appropriate numerical values, and conducted data analysis using Statwing, the statistical analysis software of Qualtrics, and Excel.

\section{Data registration and program administration}

The scale-up and retention improvement program and assessment, including data collection from the 
Table 1: Overview of metrics at beginning and follow-up.

\begin{tabular}{|l|l|l|l|}
\hline Metric & Beginning & Follow-up & P-Value \\
\hline & Sum (\%) & Sum (\%) & \\
\hline Total deliveries & & & - \\
\hline Vaginal deliveries & $8019(100 \%)$ & $7928(100 \%)$ & 0.855 \\
\hline Caesarian Sections & $6318(78.8 \%)$ & $6465(81.5 \%)$ & 0.357 \\
\hline & $1701(21.2 \%)$ & $1463(18.4 \%)$ & \\
\hline Outcome indicators & & & $0.0144^{*}$ \\
\hline Neonatal deaths $<24$ Hrs & $63(0.79 \%)$ & $25(0.32 \%)$ & $0.0013^{*}$ \\
\hline Intrapartum stillbirths & $140(1.74 \%)$ & $38(0.48 \%)$ & $0.0145^{*}$ \\
\hline Sick newborns discharged & $1205(15.0 \%)$ & $278(3.5 \%)$ & \\
\hline & & & $1785(100 \%)$ \\
\hline Total personnel trained & & $48(2.7 \%)$ & $0.0004^{*}$ \\
\hline Personnel trained & $512(28.7 \%)$ & & \\
\hline
\end{tabular}

a: Beginning was measured in Ashad, Shrawan \& Bhadra 2075; ${ }^{\text {: }}$ Follow up measurements were collected Magh, Falgun, Chaitra 2076; ": Paired t-test results with significant $p$ value.

entire cohort of facilities, was approved by the Nepal Health Research Council. The registration number is $797 / 2018$.

An SSN supervisor submitted monthly reports and periodic formal presentations to the Social Welfare Council and the National Health Research Council, both of whom had oversight responsibilities for the program. The Nepal implementation staff was composed of the program coordinator in Nepalgunj and the SSN supervisor in Kathmandu. Only the program coordinator was engaged full-time.

\section{Results}

\section{Scale-up and support}

The scale-up of training resulted in 1,785 providers receiving HBB training during the program. Approximately $29 \%$ of the healthcare personnel received HBB training in the first three months following the TOT while $3 \%$ received $\mathrm{HBB}$ training in the last three months. The new facility-based trainers, with the assistance of the program coordinator, cascaded the training in their facilities, trained new staff during the course of the program, and held refresher training sessions.

At the conclusion of the program, all facilities reported practice corners that were equipped and utilized, held periodic review meetings, conducted selfevaluation surveys, and maintained both delivery and newborn logs.

The 18 facilities in the program represented 6 different facility types: zonal hospital (2), regional hospital (3), district hospital (5), medical college (2), health posts (3), and primary health center (3) (Table 1). The urban zonal hospitals, regional hospitals, and medical colleges each accounted for about $25 \%$ of all deliveries, with the rural district hospitals, primary health centers, and health posts combining for about $25 \%$ of the total deliveries.

Medical college metrics were disproportionally greater for neonatal deaths less than 24 hours (158 or $39.5 \%$ ), intrapartum stillbirths ( 219 or $37.1 \%$ ), discharge or transfer of sick newborns $(1,821$ or $36 \%)$, and Caesarean sections (5460 or $42.6 \%$ ).

The zonal hospitals represent the highest level of care in the public system. Neonatal deaths less than 24 hours, intrapartum stillbirths, and discharge/transfer of sick newborns were disproportionately lower in these facilities.

The health posts and primary health centers did not provide Caesarean sections, yet their metrics were generally proportional to their contribution to the total number of deliveries. Outcomes according to facility type are listed in Table 2.

\section{Overview of perinatal events}

The cohort included 18 facilities. During the program, there were 49,809 vaginal deliveries and 12,823 Caesarian sections, for a total of 62,632 births included in the facility monitoring.

The total number of births assessed in the comparison between beginning and follow-up measurements was 15,947 . Analysis of this cohort suggested that the SSN program for emergency newborn care improvement was associated with decreases in the number of neonatal deaths less than 24 hours, in the number of intrapartum stillbirths, and in the number of sick newborns transferred or discharged from the maternity unit.

Table 1 summarizes the number of deliveries, personnel trained, and the change in outcome indicators in this comparison. 


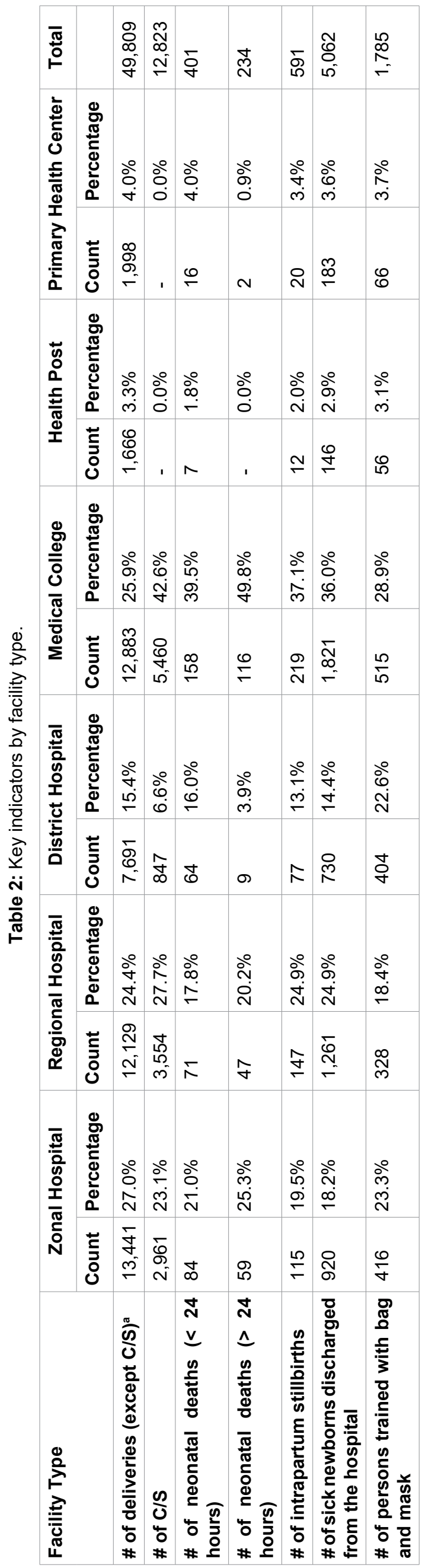

\section{Trends over time}

Neonatal deaths decreased from the beginning of the program. The average number of deaths in the first three months was 21 , compared to 8 in the last three months. This equates to a reduction of approximately $60 \%(p=0.01)$. In addition to the beginning-to-followup comparison, the trend over time in neonatal deaths is shown in Figure 1.

The number of intrapartum stillbirths generally decreased over time, as shown in Figure 2. The average number of intrapartum stillbirths decreased by approximately $73 \%(p=0.001)$, going from an average of 47 in the first 3 months to 13 in the last 3 months.

Sick newborns transferred or discharged from the maternity unit was a proxy for all-cause newborn morbidity during the first days of life. Overall, morbidity decreased over time, as shown in Figure 3 . The average number of sick newborns discharged in the first 3 months was reduced by $77 \%(p=0.01)$, causing the mean average to drop from 402 in the first three months to 93 in the last three months.

\section{Discussion}

The implementation of the Millennium Development Goals, combined with numerous influential voices (including contributors to Lancet's Newborn/Stillbirth series) appropriately refocused global attention on newborn outcomes in LMIC [26]. This newborn focus hastened the development of improved neonatal resuscitation training curricula, such as HBB, and accelerated their deployment. The Sustainable Development Goals continue to fuel this focus, with a neonatal mortality goal of 12 by 2030 [27].

HBB has been introduced, with variable scale-up, in over 90 countries. Over the last 11 years, the body of literature on the relationship between HBB and neonatal outcomes has grown considerably. HBB has been validated as effective in reducing both neonatal mortality at 24 hours and the burden of stillbirths. Mortality reductions result from the timely and competent resuscitation of severely depressed neonates. Stillbirth reductions result from the resuscitation of all nonmacerated "stillbirths," of whom portions are actually severely depressed live births.

Research has also demonstrated deterioration over time in the application and retention of new lifesaving skills [28-30]. To combat skill deterioration, the literature has provided evidence-based steps to retain skills and sustain improved newborn outcomes that are simple and effective.

Scaling-up interventions at the population level is difficult, complex, and as in many disciplines, there is a significant gap between research and implementation [31,32]. Despite numerous research protocols utilizing 


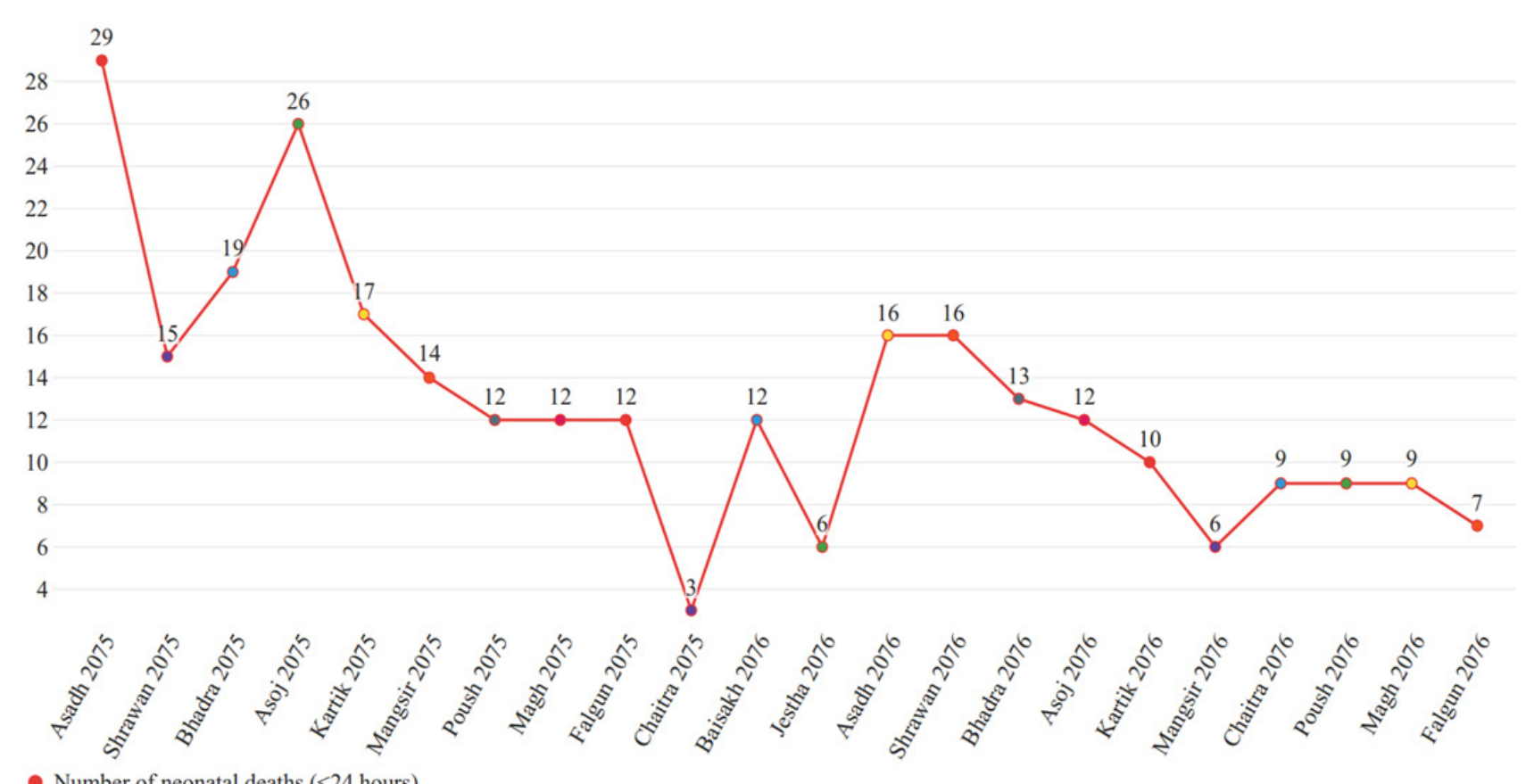

- Number of neonatal deaths ( $<24$ hours)

Figure 1: Number of neonatal deaths less than 24 hours from birth.

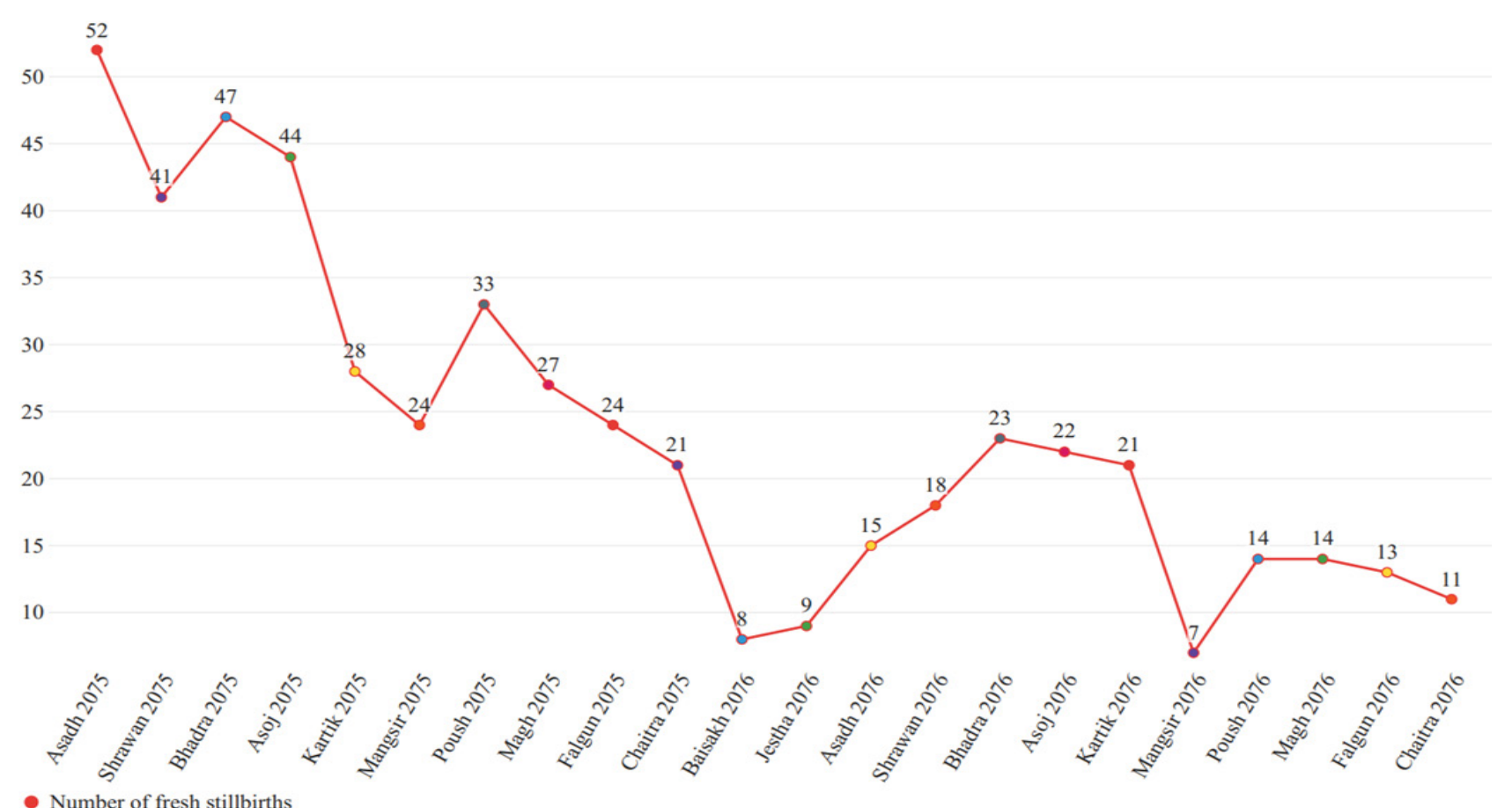

Figure 2: Number of intrapartum stillbirths.

$\mathrm{HBB}$, only very few countries have scaled up HBB nationwide. This report describes one potential model to obtain longer-term sustainability that is evidencebased, relatively low cost, and assesses limited indicators of progress. It is a model that contemplates population-level scale-up of HBB, to be modified or adjusted according to need, strengths, and challenges.

Facilities in and around Nepalgunj, Nepal, were selected due to existing disparities in newborn health outcomes. This program successfully scaled up HBB training to all newborn providers in a portfolio of 18 facilities and supported the application of improved resuscitation techniques for a period of two years. By the end of the program, all facilities were engaged in high-frequency, low-dose practice, held review meetings, conducted self-evaluation surveys, trained new staff, and held refresher training sessions.

Both the scale-up and retention efforts confronted a variety of challenges during the program that varied significantly by facility, including differences in staffing 


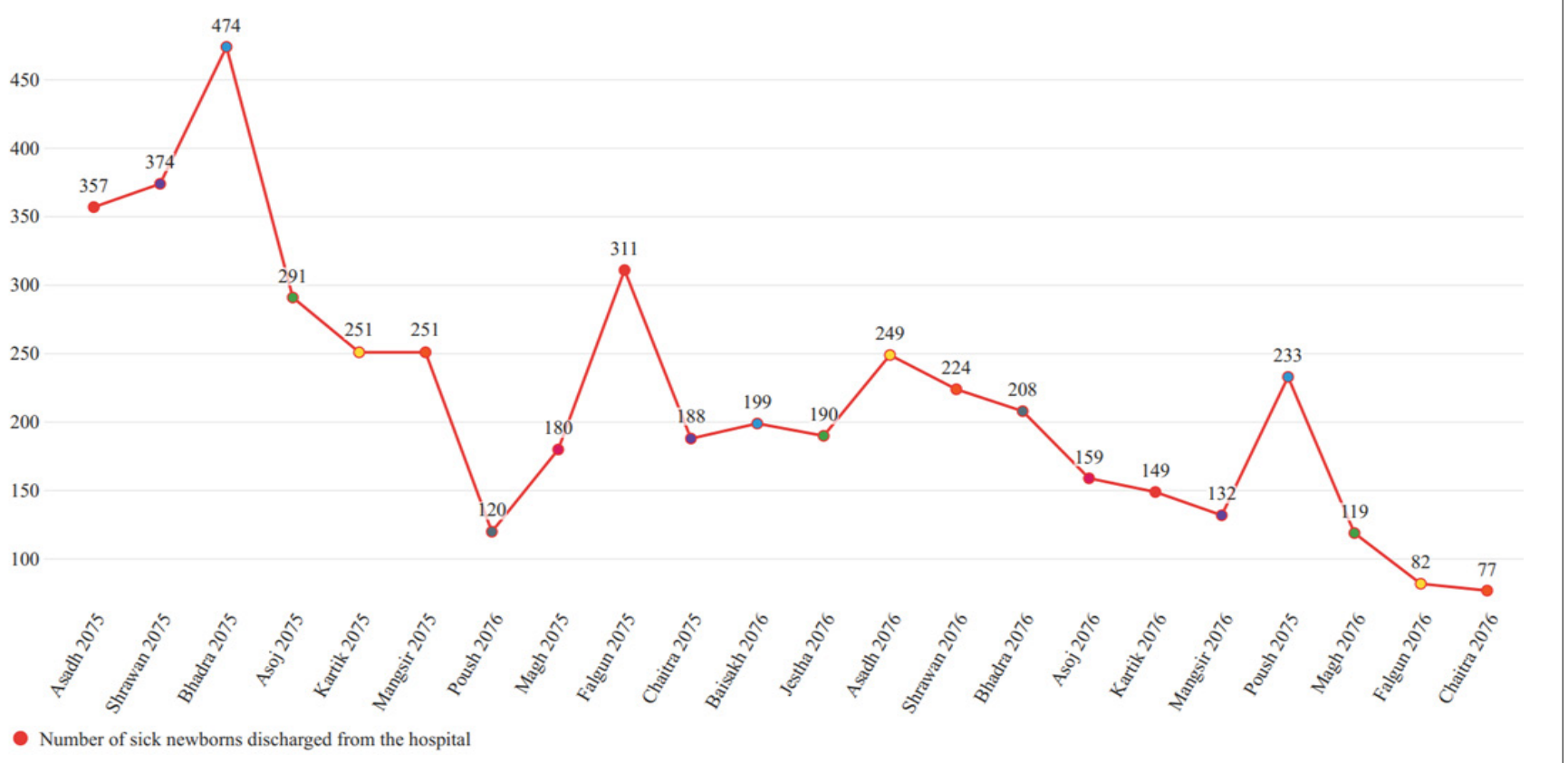

Figure 3: Number of sick newborns transferred or discharged from the maternity unit.

ratios and turnover, staff acceptance of change, and facility readiness.

The COVID-19 pandemic substantially disrupted all quality improvement efforts during the final months of the study [33]. The significant shifting of trained personnel to other hospital units resulted in the cessation of training, mentoring activities, and practice. Data collection was remote, delayed, with decreased validation of accuracy (Personal communication, Ranjan Dhungana).

Prior to the pandemic, the human resource adjustments in Ashad to Shrawan 2076 due to MOHP reorganization resulted in similar disruptions and the influx of untrained, inexperienced staff as the trained staff was transferred to other hospitals and units. This affected the overall quality of newborn care and was associated with data collection challenges (Personal communication, Ranjan Dhungana).

Throughout the program, the data collection process was not as straight forward as anticipated. Since all facilities report deaths to the MOHP, this metric was standard, tracked by the facilities, and less prone to error. Stillbirths were also tracked by most facilities prior to this program, but not always characterized by intrapartum vs. pre-admission. Accordingly, delivery logs were reviewed to obtain this information, which were influenced by intra-observer variability and bias in providers' observations and documentation. Sick newborn transfer or discharge is a proxy indicator for significant all-cause morbidity, an underreported metric in the literature. This was not a standard metric tracked by facilities and necessitated a review of newborn logs to determine the number of sick infants transferred or discharged.
As a scale-up and retention program, rather than a clinical study, there were several inherent limitations. First, demographic data of doctors, nurses, and patients were not collected. Second, there was no control group. Third, the introduction of metrics that were not routinely measured. Fourth, this program was neither intended nor designed to measure causality.

This two-year program suggests that initiatives focused on applying and maintaining HBB skills were associated with sustained improvements in newborn outcomes. 24-hour mortality decreased $60 \%$, morbidity fell $77 \%$, and intrapartum stillbirths dropped $73 \%$. While these results are encouraging, several factors may influence both the interpretation of results and the extrapolation of the results to other settings.

The improved neonatal health outcomes reported here cannot be fully attributed to the HBB scale-up program as other concurrent training programs may have contributed to the improved outcomes. HBB training had been conducted at some of the facilities by NHTC and LDSC three years previously. However, the training coordinator reported that staff at all facilities was generally HBB-naïve, illustrating the need for better retention initiatives.

The midwives, nurses, and physicians at the target hospitals may have received reinforcement of resuscitation training from other sources. Two inservice curricula, the Community-Based Integrated Management of Neonatal and Childhood IIIness (CBIMNCI) and the Skilled Birth Attendant programs have been taught intermittently in the Nepalgunj area and contain components of HBB. However, these two curricula give minimal attention to resuscitation skill development and involved only a few, if any, of the staff 
mentored in the SSN program. UNICEF clinical trials, which included HBB refreshers, did include two of the regional facilities in the Nepalgunj area [14]. Individual hospitals, such as regional facilities, may have had other resources focusing on immediate newborn care.

While the exact contribution of this HBB scale-up and support program to the marked improvements in neonatal outcomes is unknown, it may be significant. The continued scale-up of training, combined with persistent support focused on utilizing new skills coincided with steadily improving outcome measures. It is noteworthy that all three run charts showed worsening trends in Ashad 2076, consistent with the challenges of the major reassignments in healthcare personnel mentioned above (Personal communication, Ranjan Dhungana).

Strengths of the program report include the number of deliveries assessed, the range of facilities included, generally HBB-naïve facility staff, the association between shifts in the run charts and external influences, the strong support to the program from facility administrators and NHTC, and the consistency of the mentoring and supportive activities.

Newborn resuscitation training in general, and $\mathrm{HBB}$ training specifically, is not novel in Nepal. Of note is the finding that $93 \%$ of the trainers had previously used a bag and mask. The improvements associated with this program, despite prior training and experience, suggest that this approach may be appropriate for adaptation in other areas of Nepal. However, the degree of improvement may not be generalizable due to a variety of influences in other areas.

This evidence-based model, which focused on building in-facility capacity, centered on supporting infacility trainers. External resources were minimal but dedicated to this effort. Data collected were consistent with governmental standards. The program thus leveraged available evidence, current systems, and MOHP goals into a lower-cost, longer-term sustainability program than prior models.

\section{Conclusion}

The implementation and retention of neonatal life-saving skills requires support and reinforcement over time. SSN organized and supervised a two-year evidence-based, low-cost program, centered on facilitybased trainer's implementation of on-site HBB training, practice, and subsequent mentorship. This provides a valuable example of how facility-based trainers can have a substantial influence on critical neonatal outcomes. Furthermore, this program demonstrated that capacity building required minimal external support, with a single mentor supervising and monitoring quality enhancement in 18 facilities. Future research is needed to better define the sustainability of training by similar programs, strengthen the data capture of newborn parameters, measure quality gaps, and assess alternative low-cost, long-term mentoring approaches to sustainable change. Future programs working to reduce neonatal mortality, morbidity, and intrapartum stillbirths could build on the program strengths documented here to further improve neonatal outcomes.

\section{Acknowledgements}

We thank the late Rakesh Hamal, founder and director of Safa Sanaulo Nepal during this initiative, whose vision and leadership made this program possible. We are extremely grateful to the facility directors, administrators, matrons, nursing staff, and trainers for their front-line efforts to improve newborn care. The National Health Training Centers of Nepal deserve special recognition for their leadership in providing in-service education for providers across Nepal. The Nepal National Health Research Council provided an invaluable partnership with the facilities.

\section{Supporting Information}

All data underlying the findings reported herein will be available and on deposit with the Nepal National Health Research Council.

Personal Communications from Ranjan Dhungana, the field coordinator, are summarized in a letter dated 9/16/2020.

\section{Funding}

Safa Sanaulo received funding from Latter-day Saints Charities. LDSC had no role in data collection and analysis, decision to publish, or preparation of the manuscript. Volunteers for LDSC assisted SSN in the design of the scale-up/retention initiative.

\section{Conflict of Interests}

All authors report no outside financial relationships or conflicts of interest. RD and NP were full-time employees of SSN during the time of this initiative.

\section{References}

1. Commission NP (2016) Nepal and the millennium development goals. Final status report, 2000-2015. Kathmandu, Nepal: Government of Nepal, National Planning Commission.

2. Knoema (2020) Nepal neonatal mortality rate, 1960-2018.

3. UNICEF (2016) Maternal and newborn health disparities country profiles - UNICEF data.

4. Kc A, Wrammert J, Ewald U, Clark RB, Gautam J, et al. (2016) Incidence of intrapartum stillbirth and associated risk factors in tertiary care setting of Nepal: A case-control study. Reprod Health 13: 103.

5. WHO (2019) Children: improving survival and well-being.

6. UNICEF (2021) Neonatal mortality - UNICEF Data.

7. Zulliger R (2018) Nepal's frontline health workers. CHW Central.

8. Kc A, Wrammert J, Clark RB, Ewald U, Vitrakoti R, et al. 
(2016) Reducing perinatal mortality in Nepal using helping babies breathe. Pediatrics 137: e20150117.

9. Msemo G, Massawe A, Mmbando D, Rusibamayila N, Manj K, et al. (2013) Newborn mortality and fresh stillbirth rates in Tanzania after helping babies breathe training. Pediatrics 131: e353-e360.

10. Versantvoort JMD, Kleinhout MY, Ockhuijsen HDL, Bloemenkamp K, de Vries WB, et al. (2020) Helping babies breathe and its effects on intrapartum-related stillbirths and neonatal mortality in low-resource settings: A systematic review. Arch Dis Child 105: 127-133.

11. Niermeyer S (2020) Improving global newborn survival: Building upon helping babies breathe. Neonatology 117: 211-216.

12. Dol J, Campbell-Yeo M, Murphy GT, Aston M, McMillan D, et al. (2018) The impact of the Helping Babies Survive program on neonatal outcomes and health provider skills: A systematic review. JBI Database System Rev Implement Rep 16: 701-737.

13. Kc A, Ewald U, Basnet O, Gurung A, Pyakuryal SN, et. al. (2019) Effect of a scaled-up neonatal resuscitation quality improvement package on intrapartum-related mortality in Nepal: A stepped-wedge cluster randomized controlled trial. PLoS Med 16: e1002900.

14. Gurung R, Jha AK, Pyakurel S, Gurung A, Litorp $H$, et al. (2019) Scaling up safer birth bundle through quality improvement in Nepal (SUSTAIN)-a stepped wedge cluster randomized controlled trial in public hospitals. Implementation Science 14: 65.

15. Merrill RM, Frutos AM, Lyon JL, Clark RB (2020) The role of latter-day saint charities towards the establishment of needed national neonatal resuscitation programmes in resource poor countries. J Int Dev 33: 1-15.

16. Kc A, Wrammert J, Nelin V, Clark RB, Ewald U, et al. (2017) Evaluation of Helping Babies Breathe Quality Improvement Cycle (HBB-QIC) on retention of neonatal resuscitation skills six months after training in Nepal. BMC Pediatr 17: 103.

17. Drake M, Bishanga DR, Temu A, Njozi M, Thomas E, et al. (2019) Structured on-the-job training to improve retention of newborn resuscitation skills: A national cohort Helping Babies Breathe study in Tanzania. BMC Pediatr 19: 51.

18. Eblovi D, Kelly P, Afua G, Agyapong S, Dante S, et al. (2017) Retention and use of newborn resuscitation skills following a series of helping babies breathe trainings for midwives in rural Ghana. Glob Health Action 10: 1387985.

19. Tabangin ME, Josyula S, Taylor KK, Vasquez JC, KamathRayne BD (2018) Resuscitation skills after Helping Babies Breathe training: A comparison of varying practice frequency and impact on retention of skills in different types of providers. Int Health 10: 163-171.
20. Evans CL, Bazant E, Atukunda I, Williams E, Niermeyer $S$, et al. (2018) Peer-assisted learning after onsite, lowdose, high-frequency training and practice on simulators to prevent and treat postpartum hemorrhage and neonatal asphyxia: A pragmatic trial in 12 districts in Uganda. PLoS One 13: e0207909.

21. Mduma E, Kvaløy JT, Soreide E, Svensen E, Mdoe P, et al. (2019) Frequent refresher training on newborn resuscitation and potential impact on perinatal outcome over time in a rural Tanzanian hospital: An observational study. BMJ Open 9: e030572.

22. Reisman J, Arlington L, Jensen L, Louis H, Suarez-Rebling D, et al. (2016) Newborn resuscitation training in resourcelimited settings: A systematic literature review. Pediatrics 138: e20154490.

23. Thacker N, Clark R, Keenan W, et al. (2019) Nursing skill assessment for essential newborn care in one of the districts under helping babies survive pilot programme in India. Pediatric Academic Societies.

24. Wilson GM, Ame AM, Khatib MM, Khalfan BS, Thompson $J$, et al. (2020) Helping Babies Breathe (2nd edition) implementation on a shoestring budget in Zanzibar, Tanzania. Maternal Health Neonatol Perinatol 6: 3.

25. Singh S, Shrestha G, Joshi D, Gebreselassie T (2019) Childhood illness and mortality in Nepal: Trends and determinants. DHS Further Analysis Reports No. 120.

26. Lancet Series: Neonatal Survival (2005), Stillbirths (2011), Every Newborn (2014), Stillbirths (2016).

27. UNICEF (2021) Child survival and the SDGs.

28. Goudar SS, Somannavar MS, Clark R, Lockyer JM, Revankar AP, et al. (2013) Stillbirth and newborn mortality in India after helping babies breathe training. Pediatrics 131: e344-e352.

29. Bang A, Bellad R, Gisore P, Hibberd P, Patel A, et al. (2014) Implementation and evaluation of the Helping Babies Breathe curriculum in three resource limited settings: Does Helping Babies Breathe save lives? A study protocol. BMC Pregnancy Childbirth 14: 116.

30. Musafili A, Essén B, Baribwira C, Rukundo A, Persson LA (2013) Evaluating Helping Babies Breathe: Training for healthcare workers at hospitals in Rwanda. Acta Paediatrica 102: e34-e38.

31. Barker PM, Reid A, Schall MW (2015) A framework for scaling up health interventions: Lessons from large-scale improvement initiatives in Africa. Implement Sci 11: 12.

32. Westerlund A, Nilsen P, Sundberg L (2019) Implementation of implementation science knowledge: The researchpractice gap paradox. Worldviews Evid Based Nurs 16: 332-334.

33. Asish KC, Peterson SS, Gurung R, Skalkidou A, Gautam J, et al. (2021) The perfect storm: Disruptions to institutional delivery care arising from the COVID-19 pandemic in Nepal. J Glob Health 11: 05010. 